\title{
IDENTIFICATION OF A PROPOSED NEW SEROVAR OF ACTINOBACILLUS PLEUROPNEUMONIAE: SEROVAR 16
}

\author{
Rita SÁRKÖZI ${ }^{*}$, László MAKRAI and László FODOR \\ Department of Microbiology and Infectious Diseases, Faculty of Veterinary Science, \\ Szent István University, P.O. Box 22, H-1581 Budapest, Hungary
}

(Received 1 September 2015; accepted 28 October 2015)

\begin{abstract}
Five Actinobacillus pleuropneumoniae strains isolated from pathological lesions of porcine pleuropneumonia in Hungary could not be assigned to any of the accepted 15 serovars. Using hyperimmune serum raised against these untypable-serovar $A$. pleuropneumoniae strains in rabbits, indirect haemagglutination tests proved that they form a distinct group and there is no cross-reaction between them and the type strains of $A$. pleuropneumoniae. All five strains harboured the toxin-associated genes for the production (apxIA) and secretion (apxIB) of ApxI, the gene for the expression of ApxII and the largest-size $(2800 \mathrm{bp})$ apxIV gene. The carbon source utilisation pattern and the sequence analysis of the $16 \mathrm{~S}$ rRNA gene confirmed the species identification of the suggested type strain, A. pleuropneumoniae A-85/14. A new serovar of $A$. pleuropneumoniae - serovar 16 - is proposed with $A$. pleuropneumoniae A-85/14 as reference strain.
\end{abstract}

Key words: Actinobacillus pleuropneumoniae, untypable strain, new serovar, serovar 16

Actinobacillus pleuropneumoniae is one of the most important agents of the swine respiratory disease complex; it causes haemorrhagic-necrotic pneumonia and fibrinous pleuritis in growing and fattening pigs throughout the world. It is a facultative pathogenic bacterium present on the mucous membranes of the porcine respiratory tract. In the presence of predisposing factors such as overcrowding, nutritional deficiencies, management problems or bacterial and viral infections, A. pleuropneumoniae can cause severe respiratory disease and losses. The bacterium has two biotypes: biotype 1 strains need nicotinamide adenine dinucleotide (NAD, V factor) for growth, while biotype 2 strains are not NAD dependent. Several virulence factors, among others Apx toxins, fimbriae, outer membrane proteins, ability of biofilm formation, presence of transporter systems and different enzymes are involved in the pathogenesis of pneumonia caused by A. pleuropneumoniae (Chiers et al., 2010; Grasteau et al., 2011).

*Corresponding author; E-mail: sarkozi.rita@aotk.szie.hu; Phone: 0036 (1) 251-9900; Fax: 0036 (1) 251-9260 
On the basis of surface soluble capsular polysaccharide (CPS) and lipopolysaccharide (LPS) antigens, 15 serovars have been described (Nielsen, 1986a,b; Fodor et al., 1989; Nielsen et al., 1997; Blackall et al., 2002). Several methods have been used for serotyping $A$. pleuropneumoniae strains; these include slide agglutination, latex agglutination, complement fixation, indirect haemagglutination (IHA), coagglutination, ring precipitation and agar gel precipitation tests (Mittal et al., 1983a; Mittal et al., 1987; Molnár, 1990; Nielsen et al., 1997). Many authors have found that among the classical serotyping methods the IHA test is the most specific and sensitive (Mittal et al., 1983b; Nicolet et al., 1981; Nielsen and O'Connor, 1984; Nicolet, 1988). Cross-reactions make serotyping rather difficult, and such cross-reactions have been reported between serovars 1 , 9 and 11, serovars 3, 6 and 8, and serovars 4 and 7 (Mittal et al., 1988; Zhou et al., 2008). Correlation between serovars and the virulence of A. pleuropneumoniae strains was described several years ago (Marsteller and Fenwick, 1999), which can be explained by the correlation between serovars and the pattern of toxin production (Kamp et al., 1994; Frey et al., 1995). On the basis of this correlation, classical serotyping methods are nowadays frequently replaced by the detection of toxin genes; however, the latter can be misguiding in the case of strains with atypical toxin production (Rayamajhi et al., 2005; Gottschalk, 2015). More specific molecular serotyping methods detect genes involved in the biosynthesis of capsular polysaccharides (Angen et al., 2008; Bossé et al., 2014; Marois-Crehan et al., 2014; Turni et al., 2014). Using amplified fragment length polymorphism (AFLP) analysis 20 clusters could be identified, and all clusters contained strains of a single serotype (Kokotovic and Angen, 2007).

Between 2012 and 2014, a total of 50 A. pleuropneumoniae strains isolated from clinical cases from different swine farms in Hungary were serotyped by the IHA test (data not presented), but five strains could not be assigned to any of the 15 known serovars of A. pleuropneumoniae. The aim of the present study was to examine these untypable strains and to present evidence about the existence of a new serovar of A. pleuropneumoniae, serovar 16.

\section{Materials and methods}

\section{Bacteria}

Five A. pleuropneumoniae field isolates of biotype 1 were included in the present study. Two of them were isolated from the lungs of slaughtered pigs showing typical lesions of pleuropneumonia. Three strains were isolated from postmortem cases of acute porcine pleuropneumonia, submitted to our laboratory from different swine farms. The strains originated from five different farms located in different parts of Hungary, with epidemiological connection existing only between two of them (some animals were transported from one farm to the 
other). The reference strains of all 15 serovars of $A$. pleuropneumoniae were kindly provided by Dr. O. Angen (Danish Veterinary Laboratory, Copenhagen).

The A. pleuropneumoniae strains were isolated on Tryptone Soya Agar (TSA, Biolab Ltd. Budapest, Hungary) cross-inoculated with Staphylococcus aureus, and cultured on chocolate agar with added $50 \mu \mathrm{g} / \mathrm{ml}$ NAD (Biolab Ltd., Budapest, Hungary), both containing 10\% defibrinated sheep blood. Cultures were incubated at $37^{\circ} \mathrm{C}$ for $24 \mathrm{~h}$ in aerobic environment with the addition of $5 \%$ carbon dioxide. They were identified using standard methods (Barrow and Feltham, 1993). After identification, the isolated A. pleuropneumoniae strains were stored at $-80^{\circ} \mathrm{C}$ until further examination.

\section{Production of antisera}

Antisera were produced in rabbits against $A$. pleuropneumoniae reference strains of serovars 1-15 and against our five field strains as described by Biberstein (1978). Briefly, rabbits weighing about $3 \mathrm{~kg}$ were inoculated intravenously with one of the A. pleuropneumoniae strains. Bacteria from three chocolate agar cultures, incubated at $37^{\circ} \mathrm{C}$ for $24 \mathrm{~h}$, were suspended in $40 \mathrm{ml}$ saline (approximately $3 \times 10^{10}$ colony-forming units $/ \mathrm{ml}$ ) containing $0.5 \%$ formaldehyde. The suspension was allowed to stand at room temperature for $1 \mathrm{~h}$, and then the bacterial cells were pelleted by centrifugation, resuspended in saline, and stored at $4{ }^{\circ} \mathrm{C}$ until used. The rabbits were inoculated intravenously with $0.5,1,2,3,3$ and $3 \mathrm{ml}$ of the suspension, respectively, 3-4 days apart. One week after the last injection, they were bled in general anaesthesia induced with ketamine and xylazine (Animal Experiment Licence No. 22.1/2703/003/2009). Sera were harvested and stored in small aliquots in a deep freezer at $-20{ }^{\circ} \mathrm{C}$ until used.

\section{Serological characterisation}

The IHA test was performed as described by Biberstein (1978). All antisera produced against the 15 type strains of A. pleuropneumoniae and the five untypable $A$. pleuropneumoniae strains were examined with all type strains and the 5 untypable $A$. pleuropneumoniae strains. Reactions below a titre of 1:160 were neglected.

\section{Detection of toxin genes by polymerase chain reaction (PCR)}

The presence of apxIA, apxIB, apxII, apxIII and apxIV genes was examined using PCR. The primers and conditions for these PCR assays had been described by Rayamajhi et al. (2005). 


\section{Characterisation of A. pleuropneumoniae strain A-85/14}

After the serological examinations, the biochemical characteristics and the nucleotide sequence of the $16 \mathrm{~S}$ rRNA gene of a representative strain, A. pleuropneumoniae A-85/14 were determined. The utilisation of 95 carbon sources was examined using the Biolog system (BIOLOG Inc., California). The $16 \mathrm{~S}$ rRNA gene was amplified by PCR using the universal primers, its nucleotide sequence was determined as described (Relman, 1993), and the rrs gene sequence was submitted to the GenBank database (Accession No. SUB1098860 A-85/14 KT763387).

\section{Results}

Using conventional methods, all five isolates proved to be A. pleuropneumoniae biotype 1 strains. They did not show any reactions with antisera raised against the 15 recognised type strains of $A$. pleuropneumoniae in the IHA test but they reacted with their homologous sera in titres of 1:2560 and 1:5120. All the five strains gave heterologous reactions in the same titre with the hyperimmune sera raised against the other four untypable $A$. pleuropneumoniae strains. None of the 15 type strains of $A$. pleuropneumoniae gave a reaction with the sera raised against the untypable $A$. pleuropneumoniae strain A-85/14.

All five strains harboured the genes for the production (apxIA) and secretion (apxIB) of ApxI, the gene for the expression of ApxII and the largest-size (2800 bp) apxIV gene.

Based upon the utilisation of 95 carbon sources, A. pleuropneumoniae strain A-85/14 was identified as A. pleuropneumoniae, as its carbon utilisation pattern showed $99 \%$ similarity with the database. Sequence analysis of the $16 \mathrm{~S}$ rRNA detected 99\% similarity with A. pleuropneumoniae (GenBank Accession No. SUB1098860 A-85/14 KT763387).

The A. pleuropneumoniae strain A-85/14 has been deposited in the Hungarian National Collection of Medical Bacteria (HNCMB) under the registration number of 96705 .

\section{Discussion}

The five Hungarian isolates included in the present study were examined by the IHA test using hyperimmune sera raised against the 15 accepted serovars of $A$. pleuropneumoniae. As these strains did not show any reaction with the type sera, they proved to be untypable. Hyperimmune sera were produced in rabbits against the five untypable strains, and all these antisera showed both homologous and heterologous reactions of high titre within this group. None of the 15 type strains reacted with the sera produced against the untypable strains. Consequently, the five, formerly untypable A. pleuropneumoniae strains represent a 
common and distinct serovar of A. pleuropneumoniae. Untypable A. pleuropneumoniae strains can be isolated from clinical samples; some of them could be allocated in clusters together with serotypable ones indicating loss of typespecific capsule polysaccharides (Kokotovic and Angen, 2007). In the case of our strains the capsule material was present and antibodies could be raised against them, so the cause of their being untypable was not the loss of antigens. Kokotovic and Angen (2007) described two clusters containing only untypableserovar A. pleuropneumoniae strains, and they supposed that these untypable strains could represent additional serovars. Further investigations can reveal whether our strains belong to one of these clusters.

Four toxin genes, apxIA, apxIB, apxII and apxIV (2800 bp), were detected in all five strains; they can express ApxI and ApxII but not ApxIII, while ApxIV can be produced in vivo only (Schaller et al., 1999). This toxin gene pattern is typical of $A$. pleuropneumoniae serovars 5a and 5b (Rayamajhi et al., 2005). Using the IHA test, A. pleuropneumoniae serovars $5 \mathrm{a}$ and $5 \mathrm{~b}$ did not react with antisera produced against the five untypable strains, so they do not belong to serovar $5 \mathrm{a}$ or $5 \mathrm{~b}$. The discrepancy between the results of the IHA test and serotyping based on the detection of toxin genes can be explained by the fact that the latter method does not detect genes responsible for antigen production; rather, it is based on the correlation between certain serovars and toxin production of the $A$. pleuropneumoniae strains. Although new molecular methods are widely used for serotyping A. pleuropneumoniae (Rayamajhi et al., 2005; Bossé et al., 2014; Marois-Crehan et al., 2014; Turni et al., 2014; Gottschalk, 2015), they cannot always replace traditional serotyping tests.

The results of utilisation of 95 carbon sources and the sequence analysis of the 16S rRNA gene confirmed that the representative A. pleuropneumoniae A85/14 strain is a typical A. pleuropneumoniae.

Among the five A. pleuropneumoniae biotype 1 strains which proved to be untypable using hyperimmune sera raised against the 15 type strains of $\mathrm{A}$. pleuropneumoniae, a new serovar was identified, represented by strain A-85/14, and all the five untypable $A$. pleuropneumoniae strains of our collection belong to this serovar. This serovar can be clearly differentiated from the other 15 serovars of $A$. pleuropneumoniae by IHA.

In conclusion, this newly characterised serovar represents a distinct serovar, and we recommend that it be designated as serovar 16 of A. pleuropneumoniae with $A$. pleuropneumoniae A-85/14 strain as the type strain. The recommended type strain has been deposited in the HNCMB under the accession number of 96705 , and its $r$ rs gene sequence has been submitted to GenBank (Accession No. SUB1098860 A-85/14 KT763387). 


\section{Acknowledgement}

This work was supported by the Hungarian Scientific Research Fund (OTKA 84220 and OTKA 112826).

\section{References}

Angen, Ø., Ahrens, P. and Jessing, S. G. (2008): Development of multiplex PCR test for identification of Actinobacillus pleuropneumoniae serovars 1, 7 and 12. Vet. Microbiol. 132, 312-318.

Barrow, G. I. and Feltham, R. K. A. (eds) (1993): Cowan and Steel's Manual for the Identification of Medical Bacteria. Cambridge University Press, Cambridge. 331 pp.

Biberstein, E. L. (1978): Biotyping and serotyping of Pasteurella haemolytica. In: Bergan, T. and Norris, J. R. (eds) Methods in Microbiology 10. Academic Press, London. pp. 253-269.

Blackall, P. J., Klaasen, H. L. B. M., Bosch, H. V. D., Kuhnert, P. and Frey, J. (2002): Proposal of a new serovar of Actinobacillus pleuropneumoniae: serovar 15. Vet. Microbiol. 84, 47-52.

Bossé, J. T., Li, Y., Angen, O., Weinert, L. A., Chaudhuri, R. R., Holden, M. T., Williamson, S. M., Maskell, D. J., Tucker, A. W., Wren, B. W., Rycroft, A. N. and Langford, P. R. (2014): Multiplex PCR assay for unequivocal differentiation of Actinobacillus pleuropneumoniae serovars 1 to 3, 5 to 8, 10 and 12. J. Clin. Microbiol. 52, 2380-2385.

Chiers, K., de Waele, T., Pasmans, F., Ducatelle, R. and Haesebrouck, F. (2010): Virulence factors of Actinobacillus pleuropneumoniae involved in colonisation, persistence and induction of lesions in its porcine host. Vet. Res. 41, 65.

Fodor, L., Varga, J., Molnár, É. and Hajtós, I. (1989): Biochemical and serological properties of Actinobacillus pleuropneumoniae biotype 2 strains isolated from swine. Vet. Microbiol. 20, 173-180.

Frey, J., Beck, M., Bosch, J. V. D., Segers, R. P. A. M. and Nicolet, J. (1995): Development of an efficient PCR method for toxin typing of Actinobacillus pleuropneumoniae strains. Mol. Cell Probes 9, 277-282.

Gottschalk, M. (2015): The challenge of detecting herds sub-clinically infected with Actinobacillus pleuropneumoniae. Vet. J. 206, 30-38. doi:10.1016/j.tvj1.2015.06.016

Grasteau, A., Tremblay, Y. D. N., Labrie, J. and Jacques, M. (2011): Novel genes associated with biofilm formation of Actinobacillus pleuropneumoniae. Vet. Microbiol. 153, 134-143.

Kamp, E. M., Vermeulen, T. M. M., Smits, M. A. and Jaagsma, J. (1994): Production of Apx toxins by field strains of Actinobacillus pleuropneumoniae and Actinobacillus suis. Infect. Immun. 62, 4063-4065.

Kokotovic, B. and Angen, O. (2007): Genetic diversity of Actinobacillus pneumoniae assessed by amplified fragment length polymorphism analysis. J. Clin. Microbiol. 45, 3921-3929.

Marois-Crehan, C., Lacouture, S., Jacques, M., Fittipaldi, N., Kobisch, M. and Gottschalk, M. (2014): Development of two real-time polymerase chain reaction assays to detect Actinobacillus pleuropneumoniae serovars 1-9-11 and serovar 2. J. Vet. Diag. Invest. 26, 146-149.

Marsteller, T. A. and Fenwick, B. (1999): Actinobacillus pleuropneumoniae disease and serology. Swine Health Prod. 7, 161-165.

Mittal, K. R., Higgins, R. and Lariviére, S. (1983a): Detection of type-specific antigens in the lungs of Haemophilus pleuropneumoniae-infected pigs by coagglutination test. J. Clin. Microbiol. 18, 1355-1357.

Mittal, K. R., Higgins, R. and Lariviére, S. (1983b): Determination of antigenic specificity and relationship among Haemophilus pleuropneumoniae serotypes by an indirect hemagglutination test. J. Clin. Microbiol. 17, 787-790. 
Mittal, K. R., Higgins, R. and Lariviére, S. (1987): An evaluation of agglutination and coagglutination techniques for serotyping Haemophilus pleuropneumoniae isolates. Am. J. Vet. Res. 48, 219-226.

Mittal, K. R., Higgins, R. and Lariviére, S. (1988): Serologic studies of Actinobacillus (Haemophilus) pleuropneumoniae strains of serotype- 3 and their antigenic relationships with other $A$. pleuropneumoniae serotypes in swine. J. Vet. Res. 49, 152-155.

Molnár, É. (1990): Survey of Actinobacillus (Haemophilus) pleuropneumoniae infection in swine by different methods. Acta Vet. Hung. 38, 231-238.

Nicolet, J. (1988): Taxonomy and serological identification of Actinobacillus pleuropneumoniae. Can. Vet. J. 29, 578-580.

Nicolet, J., Paroz, P., Krawinkler, M. and Baumgartner, A. (1981): An enzyme-linked immunosorbent assay, using an EDTA-extracted antigen for the serology of Haemophilus pleuropneumoniae. Am. J. Vet. Res. 42, 2139-2142.

Nielsen, R. (1986a): Serology of Haemophilus (Actinobacillus) pleuropneumoniae serotype 5 strains: establishment of subtypes A and B. Acta Vet. Scand. 27, 49-58.

Nielsen, R. (1986b): Serological characterization of Actinobacillus pleuropneumoniae strains and proposal of a new serotype: serotype 12. Acta Vet. Scand. 27, 453-455.

Nielsen, R. and O'Connor, P. J. (1984): Serological characterization of 8 Haemophilus pleuropneumoniae strains and proposal of a new serotype: serotype 8. Acta Vet. Scand. 25, 96-106.

Nielsen, R., Andresen, L. O., Plambeck, T., Nielsen, J. P., Krarup, L. T. and Jorsal, S. E. (1997): Serological characterization of Actinobacillus pleuropneumoniae biotype 2 strains isolated from pigs in two Danish herds. Vet. Microbiol. 54, 35-46.

Rayamajhi, N., Shin, S. J., Kang, S. G., Lee, D. Y., Ahn, J. M. and Yoo, H. S. (2005): Development and use of a multiplex polymerase chain reaction assay based on Apx toxin genes for genotyping of Actinobacillus pleuropneumoniae isolates. J. Vet. Diagn. Invest. 17, 359-362.

Relman, D. A. (1993): Universal bacterial 16S rDNA amplification and sequencing. In: Pershing, D. H., Smith, T. F., Tenover, F. C. and White, T. J. (eds) Diagnostic Molecular Microbiology: Principles and Applications. American Society for Microbiology, Washington, D.C. pp. 489-495.

Schaller, A., Kuhn, R., Kuhnert, P., Nicolet, J., Anderson, T. J., MacInnes, J. I., Segers, R. P. A. M. and Frey, J. (1999): Characterization of apxIVA, a new RTX determinant of Actinobacillus pleuropneumoniae. Microbiology 145, 2105-2116.

Turni, C., Singh, R., Schembri, M. A. and Blackall, P. J. (2014): Evaluation of a multiplex PCR to identify and serotype Actinobacillus pleuropneumoniae serovars 1, 5, 7, 12 and 15. Lett. Appl. Microbiol. 59, 362-369.

Zhou, L., Jones, S. C. P., Angen, Ø., Bossé, J. T., Nash, J. H. E., Frey, J., Zhou, R., Chen, H. C., Kroll, J. S., Rycroft, A. N. and Langford, P. R. (2008): Multiplex PCR that can distinguish between immunologically cross-reactive serovars 3, 6, and 8 Actinobacillus pleuropneumoniae strains. J. Clin. Microbiol. 46, 800-803. 